\title{
Emerging neo adjuvants for harnessing therapeutic potential of M1 tumor associated macrophages (TAM) against solid tumors: Enusage of plasticity
}

\author{
Vinod Nadella ${ }^{1}$, Manoj Garg ${ }^{2}$, Sonia Kapoor ${ }^{2}$, Tuhar Singh Barwal ${ }^{3}$, Aklank Jain ${ }^{3}$, Hridayesh Prakash ${ }^{4}$ \\ ${ }^{1}$ Laboratory of Translational Medicine, School of Life Sciences, University of Hyderabad, Telangana, India; ${ }^{2}$ Amity Institute of Molecular Medicine \\ and Stem cell Research, Amity University Uttar Pradesh, Sector 125, Noida, India; ${ }^{3}$ Department of Zoology, Central University of Punjab, \\ Bhatinda, India; ${ }^{4}$ Amity Institute of Virology and Immunology, Amity University Uttar Pradesh, Sector 125, Noida, India \\ Contributions: (I) Conception and design: H Prakash; (II) Administrative support: H Prakash; (III) Provision of study materials or patients: None; \\ (IV) Collection and assembly of data: None; (V) Data analysis and interpretation: None; (VI) Manuscript writing: All authors; (VII) Final approval of \\ manuscript: All authors. \\ Correspondence to: Dr. Hridayesh Prakash. Amity Institute of Virology and Immunology, Amity University, Sector 125, NOIDA, Uttar Pradesh, India. \\ Email: hprakash@amity.edu.
}

\begin{abstract}
Macrophages are a major component of the tumor microenvironment (TME) of most tumors. They are characterized by a high degree of functional plasticity which enable these cells to both promote and eliminate established tumors. Under the influence of immunosuppressive TME, tumor infiltrating iNOS+ and CD11b+ M-1 effector macrophages get polarized towards tumor associated macrophages (TAM) which are tropic to variety of tumors. Increased infiltration and density of TAM is associated with tumor progression and poor prognosis in the plethora of tumors due to their angiogenetic and tissue re-modelling nature. Importantly, TAMs are also responsible for developing endothelium anergy, a major physical barrier for majority of cancer directed immune/chemotherapies. Therefore, functional retuning/re-educating TAM to $\mathrm{M}-1$ phenotypic macrophages is paramount for effective immunotherapy against established tumors. In this review, we discuss and provide comprehensive update on TAM-targeted approaches for enhancing immunity against various solid tumors.
\end{abstract}

Keywords: Tumor-associated macrophages (TAM); tumor microenvironment (TME); macrophage polarization; nanocarriers; nanoparticles; radiotherapy; small molecules inhibitors; epigenetic; tumor immune modulation; immunotherapy

Submitted Jan 14, 2020. Accepted for publication Jun 03, 2020.

doi: 10.21037/atm-20-695

View this article at: http://dx.doi.org/10.21037/atm-20-695

\section{Introduction}

Over the past decades, considerable advances were seen in the way cancer was diagnosed and treated. With advanced proteomic, genomic and bioinformatic approaches, several regulatory elements that are responsible for the manifestation of the cancerous phenotype were identified and targeted. Despite enough advancements in the field, there exists few success stories as far as the treatment of cancer is concerned. This is mainly due to the refractory nature of the multifaced immunosuppressive tumor microenvironment (TME) $(1,2)$, a complex ecosystem that is rich in heterogeneous population of stromal cells, extracellular matrix, lymphatics, and highly active innate and adaptive immune cells $(3,4)$. Of which, macrophages, called the tumor associated macrophages (TAM) are one of most abundant cell types of the leukocyte infiltrate of the TME, whose accumulation has been considered to associate with poor prognosis in most solid tumors (5-7). 


\section{TAM ontogeny and function}

Macrophages are an important part of immune system and are found in all tissues. They execute a broad spectrum of functions ranging from modulating tissue homeostasis, defense against microbial insults and facilitating wound healing (8). TAMs originate from both yolk sac derived macrophages and from monocyte precursors that are originated in the bone marrow. While the former are embryonically seeded in tissue and maintained through selfrenewal, the latter infiltrate the tissue in response to the tissue specific chemokine signals and differentiate in response to their microenvironment (9). Macrophages are extremely plastic in nature displaying multiple polarization states which fit within two most accepted extreme phenotypes: the M1-like or proinflammatory phenotype and the M2like or anti-inflammatory (10). Toll like receptor legends like lipopolysaccharides (LPS), Th1 cytokines such as Interferon gamma (IFN $\gamma)$, Tumor necrotic factor alpha (TNF $\alpha)$, interleukin-12 (IL-12), IL-18 promote macrophages towards M1 polarized state which play critical role in innate host defense (11). On the other hand, Th2 cytokines such as IL-4, IL-10, IL-13 tune macrophages towards M2-like phenotype that display anti-inflammatory responses such as tissue repair and wound healing (12). M1-like phenotypic macrophages mount a robust anti-tumoral response and support adaptive immune responses by presenting tumor-specific antigens and by producing cytokines and chemokines that recruit and activate cytotoxic $\mathrm{CD}^{+} \mathrm{T}$ cells and NK cells and suppress lethal tumor development (13). Several studies have also highlighted the role of cytotoxic lymphocytes in tumors correlating with favorable prognosis $(14,15)$. However, the plasticity nature of the macrophages allows tumor cells to subvert the macrophage function by the cues within TME and elicit an M2-like polarization that is pro-tumorigenic (16). M2-like macrophages in the TME secret immunosuppressive molecules including IL-10, arginase-1 (Arg-1), programmed death-ligand-1 (PD-L1), TGF $\beta$ and VEGF favoring tumor growth and NO $(17,18)$. TAM also secrete various chemokines such as CCL4, CCL5, CCL22 and CCL28 that attract regulatory T cells to infiltrate into the TME, thereby impeding cytotoxic T cell activation (19) suggesting that under influence of Th2 rich microenvironment of tumor, the infiltrating M1 effector macrophages and $\mathrm{T}$ cell get polarized towards M2 and regulatory $\mathrm{T}$ cells which promote tumor cell growth.

Pathologically, cancers are classified nearly into 200 types with varying degree of resistance against existing anti-cancer drug/therapies (20). Among various reasons, inefficient $\mathrm{T}$ cell migration is a major limitation of cancer immunotherapy $(15,21)$. This is mainly due to increased infiltration of macrophages and their association with tumor cells which drives desmoplastic reactions during the course of tumorigenesis. Once accumulated, TAM secretes various angiogenesis factors, such as vascular endothelial growth factor (VEGF), platelet-derived growth factor, proangiogenic growth factor (PDGF) and transforming growth factor beta (TGF $\beta$ ) within TME which serve as prognostic factors for cancer progression. VEGF has long been considered a strong pro-tumorigenic factor and anti-VEGF antibodies have shown promising antitumoral outcomes (22). M2-like macrophage polarization is induced by Colony stimulating factor 1 (CSF1) and it was shown that inhibiting CSF1 displayed significant attenuation in angiogenic programming as a result of TAM depletion coupled with tumor burden (23). Moreover, hypoxic or anoxic micro-environment of solid tumor poses a challenge to traditional cancer treatment which is mainly due to increased angiogenesis of tumor. TAM also express angiopoietin receptor, endothelial-specific receptor tyrosine kinase TIE2 and conditional knockdown of TIE2 gene was found sufficient enough to inhibit tumor angiogenesis (24). Pro-angiogenic functions of TAM are also facilitated by the hypoxia-inducible factor 1 alpha (HIF1- $\alpha$ ) which transcriptionally upregulate VEGF expression $(25,26)$. This renders the tumor endothelium impermissive for cytotoxic $\mathrm{T}$ cells infiltration while predisposing tumor cells less susceptible to death by various chemotherapeutic drugs and/or radiotherapy which aimed to increased intratumoral oxygenation for promoting immunogenic death of tumor cells.

Tumor metastasis is responsible for $90 \%$ of cancer-related mortality and TAM play a prominent role in facilitating tumor invasion and metastasis by secreting several molecules such as matrix metalloproteinases, cathepsins, serine proteases, CCL2, CCL18, and cyclooxygenases. Wei et al. have shown that TAM-derived IL6induce epithelialmesenchymal transition (EMT) program to enhance human colorectal cancer migration, invasion and Circulating tumor cell- mediated metastasis by regulating the JAK2/STAT3/ miR-506-3p/FoxQ1 axis leading to the production of CCL2 that promote macrophage migration (27). Steenbrugge et al. have shown that levels of MMP-9, VEGF, chitinase-3like protein 1 (CHI3L1) and Lipocalin-2 (LCN2) induced by TAMs mediate cancer metastasis in mouse model for triple-negative breast cancer (28). It was also shown that 
TAM enhance tumor cell migration and invasion through a paracrine loop consisting of macrophage-derived epidermal growth factor receptor and CSF-1 and genetic abalation of CSF-1 significantly minimized the circulating tumor cells and their metastasis (29). Therapeutic potential of S100A8 and S100A9 that were upregulated by TAM was also evaluated for interference of metastasis (30).

\section{Potential and emerging neo adjuvant for Retuning of TAM}

The hypoxic or anoxic micro-environment of solid tumor coupled with increased angiogenesis poses a challenge to traditional cancer treatment options such as chemotherapy and radiotherapy as these conditions predisposes tumor cells less susceptible to death induced by various chemotherapeutic drugs and/or radiation $(25,31)$. The presence of TAM with in the TME is the hallmarks of cancer-associated inflammation as TAM found to be a major cause and player in inducing conditions in favor of tumor survival. Therefore, depletion of macrophages will be a reliable strategy to mitigate TAM mediated tumor survival and progression (32). Several studies over the past had highlighted the possible antitumor outcomes as a means of TAM depletion. Importantly, as the growth factor CSF-1 is known to involved in the proliferation, differentiation and survival of macrophages, targeting CSF-1/CSF-1R axis was found to be an attractive target to reduce the number of TAMs in tumors. Use of emactuzumab, a humanized mAb targeting CSF-1R has shown to decrease TAM numbers in the tumor (33). Pharmacological targeting of CSF-1R was also found effective to deplete TAMs and to restore $\mathrm{T}$ cell migration into the TME of mouse mammary tumor models (34). Macrophage depletion using antibodies against F4/80 was also found to reduce the tumor growth (35). Similarly, Clodronate liposomes mediated macrophage depletion was also found promising in mitigating tumorigenesis $(36,37)$. Trabectedin, a natural product derived from marine organism Ecteinascidia turbinate has been explored for depleting macrophages on account of its ability to inactivate NF-Y, and KLF-2/4 transcription factors which are important for differentiation of mononuclear phagocyte in TME (38). In similar lines, targeting non canonical NF$\kappa B$, STAT3 and HIF-1 also represent another potential therapeutic option for dampening TAM activities in neo adjuvant settings. Although, macrophage depletion strategies found to have a positive impact by limiting tumor progression, there are several pieces of information highlighting the possible toxic effects associated which not only impact the quality of life of cancer-treated patients but also limits the translational relevance of these studies. As TAM mediated tumorigenesis is prompted by immunological imbalance in M1/M2 rheostat, enrichment or stabilization of M1 effector phenotype or re-tuning the pro-tumorigenic M2-like phenotypic macrophages towards anti-tumorigenic M1-like phenotypic macrophages would be one of the promising interventions for tumor control. Therefore, several pre-clinal and clinical studies were focused at targeting molecules that aid that functionally reprogram TAMs towards anti-tumorigenic phenotype (Table 1). In this context and within scope of this article here we discuss few strategies which have been explored by us and others having M1 polarization potential in various models.

\section{Low dose radiation (LDR) based immunotherapy}

The efficacy of conventional radiation therapy, which is one of the most widely used treatment options for cancer treatment, is limited by resistance of tumors, most importantly by associated toxicity to the normal tissue and result in immune suppression. On the contrary, LDR modulates a variety of immune responses particularly in the context of cancer. Our pre-clinical as well as clinical studies have demonstrated the therapeutic advantage of LDR in enhancing infiltration of tumor antigen specific $T$ cell and subsequent tumor immune rejection of pancreatic cancer in new adjuvant setting in a macrophage dependent manner (15). Exposure to LDR enhanced the infiltration of CD11b+ macrophages along with adoptively transferred $\mathrm{T}$ cell in the tumor of Rip Tag 5 mice. LDR exposure also facilitated spontaneous infiltration of host $\mathrm{T}$ cells, favoring tumor infiltration of $\mathrm{CD}^{+} \mathrm{T}$ cells overCD $4^{+}$ T-cells demonstrating the therapeutic efficacy of LDR for enhancing anti-tumor immunity in these mice. Interestingly, we found that LDR in conjugation with adaptive transfer of $T$ cells triggered vasculature normalization as indicated by expression of CD31 and VCAM-1, which is a prerequisite of leukocyte recruitment into tumor tissue and $\mathrm{T}$ cells infiltration coupled with co-infiltration of CD11b+ macrophages. However, depletion of macrophages with clodronate loaded liposome significantly abolished LDR mediated tumor infiltration of tumor directed $\mathrm{T}$ cell and reduced blood glucose levels predisposing mice sensitive for death suggesting the indispensable role of peritumoral macrophages in $\mathrm{T}$ cell recruitment and in tumor 
Table 1 Pre-clinical and clinical trials targeted at TAM reprogramming

\begin{tabular}{|c|c|c|c|c|c|}
\hline Clinical trail & TAM targeting agent & Target & $\begin{array}{l}\text { Clinical } \\
\text { phase }\end{array}$ & Status & Tumor type \\
\hline NCT03123783 & APX005M & CD40 & $\mathrm{I} / \mathrm{II}$ & Active & Non-small cell lung cancer; metastatic melanoma \\
\hline NCT02304393 & Selicrelumab & CD40 & I & Active & Advanced or metastatic solid tumors \\
\hline NCT01433172 & $\begin{array}{l}\text { (GM.CD40L) vaccine with } \\
\text { CCL21 }\end{array}$ & CD40 & $\mathrm{I} / \mathrm{II}$ & Active & Lung cancer \\
\hline NCT02890368 & TTI-621 & SIRP $\alpha-\lg G 1 \mathrm{Fc}$ & I & Active & $\begin{array}{l}\text { Solid tumors; breast carcinoma melanoma; } \\
\text { Merkel-cell carcinoma; squamous cell carcinoma }\end{array}$ \\
\hline NCT03530683 & TTI-622 & $\mathrm{SIRP} \alpha-\lg G 4 \mathrm{Fc}$ & I & Active & Lymphoma; myeloma \\
\hline NCT01839604 & AZD9150 (ISIS-STAT3Rx) & STAT3 & $\mathrm{l} / \mathrm{lb}$ & Completed & Advanced/metastatic hepatocellular carcinoma \\
\hline NCT00912327 & $\beta$-glucan & MAPK & II & Completed & Stage IV KRAS-mutant colorectal cancer \\
\hline NCT03435640 & NKTR262 & TLR7/8 & $\mathrm{I} / \mathrm{II}$ & Active & $\begin{array}{l}\text { Melanoma; Merkel cell carcinoma; breast cancer; } \\
\text { renal cell carcinoma; colorectal cancer }\end{array}$ \\
\hline NCT03262103 & $\begin{array}{l}\text { Polyinosinic-polycytidylic } \\
\text { acid (Poly ICLC) }\end{array}$ & TLR3 & $\mathrm{I} / \mathrm{II}$ & Active & Solid tumors \\
\hline NCT03681951 & GSK3145095 & RIP & $\mathrm{I} / \mathrm{II}$ & Active & Neoplasms; pancreatic \\
\hline NCT02637531 & IPI-549 & $\mathrm{PI} 3 \mathrm{~K} \gamma$ & I & Active & $\begin{array}{l}\text { Advanced solid tumor; non-small cell lung cancer; } \\
\text { melanoma; breast cancer }\end{array}$ \\
\hline
\end{tabular}

metabolism.

Most interestingly, adoptive transfer of Rip Tag5 mice with irradiated macrophages (M1 macrophages) alone potentially enhanced cytotoxic $\mathrm{CD}^{+} \mathrm{T}$ cells infiltration, normalized tumor vasculature, reduced hemorrhage, and efficiently caused tumor rejection thus demonstrating the need for TAM retuning. Most interestingly, the extent of $\mathrm{T}$ cell infiltration remained comparable in the animals which either received irradiated macrophages or external irradiation suggesting that adoptive transfer of iNOS+ macrophages may be a good alternate of radiotherapy. Most interestingly adoptive transfer with iNOS+ macrophages completely depleted or replaced intra tumoral MDSC populations which further added to overall M1 retuning of these tumors by M1 macrophages.

In contrast to unirradiated population of peritoneal macrophages which expressed M2-associated transcription factors and minor amounts of iNOS, local tumor irradiation induced iNOS expression and acquisition of an M1 phenotype. Indeed, in vitro irradiation of isolated RT5 peritoneal macrophages and total body irradiation in peritoneal macrophages also induced iNOS expression demonstrating that LDR induces iNOS expression and increases $\mathrm{NO}$ in CD11b+ tumor infiltrating macrophages during adoptive $\mathrm{T}$ cell transfer. Moreover, we found that inhibiting iNOS completely inhibited endothelial cell activation and Tumor infiltration of T cells emphasizing the involvement of iNOS + M1 phenotypic macrophages. Our clinical trial data with patients with advanced pancreatic adenocarcinoma demonstrated translational and therapeutic impact of locally applied gamma irradiation in triggering tumor infiltration of M1 and effector T cells in the clinical trial launched with stage $2^{\text {nd }} / 3^{\text {rd }}$ stage (as per IUCC criteria) PDAC patients as well (15). Indeed, irradiated RipTag 5 mice showed significantly higher expression of iNOS both in their TAM as well as in the insulinoma over sham irradiated mice. LDR moreover retuned TME of insulinoma which was confirmed by spontaneous infiltration 


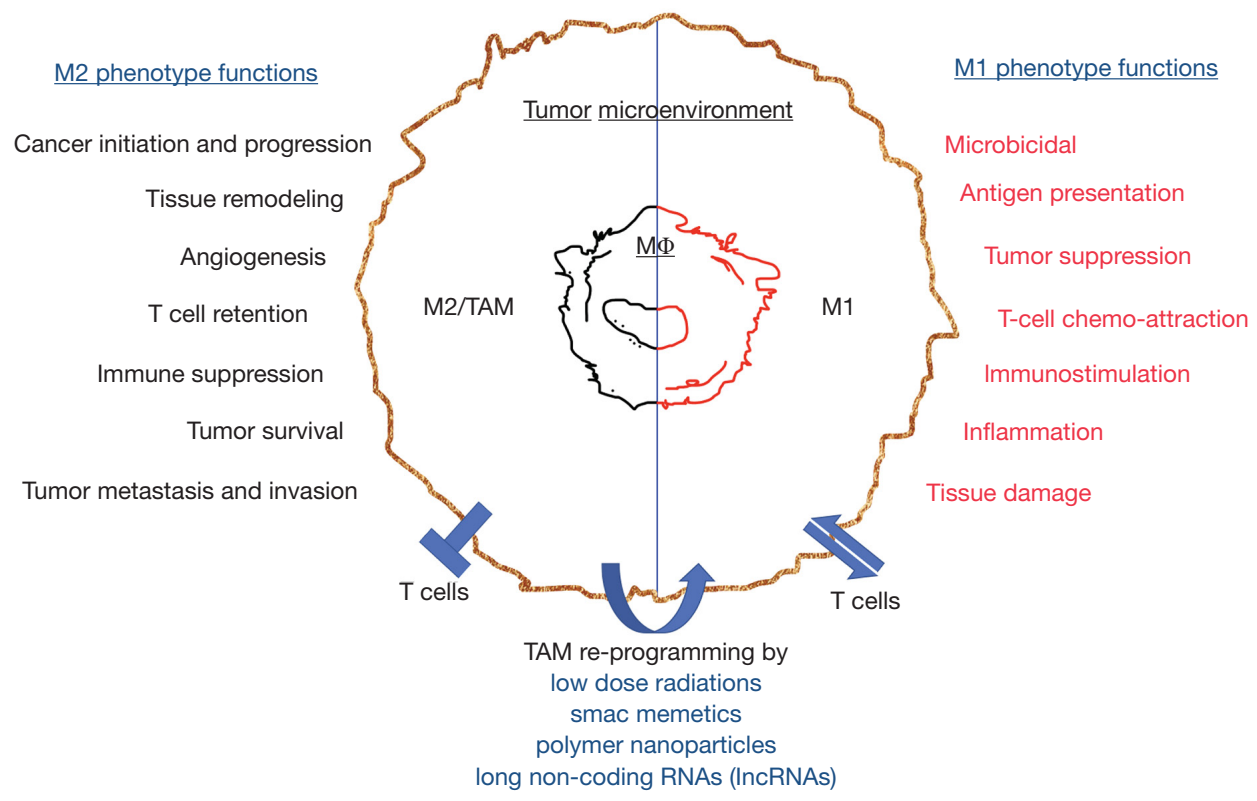

Figure 1 Disruption of functional plasticity of tumor associated macrophages by various neo adjuvants for affording immunity against cancer. Macrophages are the special cells of host immune system, which, by their virtue of phenotypic plasticity can both control as well promote tumor growth thus represent key therapeutic target of current and future cancer immunotherapeutic interventions.

of $\mathrm{F} 4 / 80+$, iNOS+, CD14 and CD68 positive and classically activated $\mathrm{M} 1$ macrophages and concomitant reduction of Ym-1, Fizz-1, Arginase-1 and CD206 expressing M2-like macrophages in these mice. LDR also induced expression of iNOS, NO, NFкB pp65, pSTAT3 and proinflammatory cytokines secretion while downregulating p38MAPK which are involved in iNOS translation and acquisition of an M1-like phenotype (39). LDR down-modulated HIF-1 and VEGF-induced angiogenic signaling in tumor-derived endothelial cells in irradiated tumors in the course of polarization of irradiated tumor-associated macrophages toward an M1 phenotype (40). Most interestingly, depletion of macrophages for prolonged period of time potentially inhibited gamma irradiation-induced iNOS+ macrophages and enhanced expression levels of M2 markers including Arginase-1, Ym-1 and Fizz-1 along enhanced key immunosuppressive cytokines including VEGF, TGF- $\beta$ which otherwise were significantly inhibited by gamma irradiation in these tumors. In similar lines, Liu et al. have shown that use of combination of LDR and hypo-fractioned radiation therapy in a syngeneic mouse model of breast and colon carcinoma potentially enhanced systemic immune related response of radiation therapy by infiltration of $\mathrm{CD} 8^{+}$ $\mathrm{T}$ cells and altered the immune suppressive tumor micro environment (41). Interestingly, LDR was also found to increase systemic response rate of metastatic disease treated with high-dose radiation (42). Use of LDR therapy in combination with chemotherapy was found to potentiate the effects of paclitaxel and carboplatin in locally advanced squamous cell cancer of the head and neck $(43,44)$. These observations potentiate the use and involvement of LDR in tuning TAM towards M1-like proinflammatory macrophage phenotype that is anti-tumorigenic (Figure 1).

\section{PAMP based retuning of TAM population and Immunotherapy of pancreatic tumors}

Effective immunotherapy should impact anti-tumor potential in M1 repolarized TAM population in solid tumors. Many conserved bacterial ligands, the agonists for innate immune system receptors such as toll-like receptors, upon binding, initiate an intracellular signaling cascade leading to the production of proinflammatory cytokines and initiate anti-tumor activities not only by activating immune cells but also acting directly on tumor cells. In past, various TLR agonists like CpG, CD40L having potential of retuning M2 TAM to M1 macrophages have been explored (45-47). However, the success rate remained minimal due to the fact that activation of TLR's preferentially on tumor cells shown to induce angiogenesis and tumor growth (48). 
It was evident that during therapy, various TLR agonist like heparan sulphates, heat shock proteins, High Mobility Group Box 1 (HMGB1), hyaluronic acid released from the dying tumor cells activate TLR's on other intact tumor cells within TME. Subsequent binding of these DAMP's to their respective TLR preferentially on tumor cells trigger tumor cell proliferation and associated refractory immune response which is immunosuppressive (49). Although these TLR ligands can interact with both macrophages and tumor cells, due to abundance of tumor cells over TAM, TLR signaling mainly activate tumor cells. Therefore, use of macrophage specific TLRs for selective activation is anticipated to be detrimental for tumor cells (50). To this purpose, we have explored the significance of LPS as abacterial adjuvant for activating TLR4 signaling in macrophage tumor cells co-cultures and demonstrated that stimulation with LPS alone or in combination with IFN- $\gamma$ not only stabilized NO response in tumor induced macrophages but also imparted anti-tumor potential (39). Our results even justified the criteria of using LPS as TLR4 ligand for priming TAM for acquiring M1 phenotype as well as anti-tumor potential. LPS also utilizes CD14 receptors for skewing Th1/Th17 responses in macrophages and contribute for anti-tumor potential of reprogrammed TAM. Similarly, activation of TLR4/CD14 complex is known to activate mitogen-activated protein kinase (MAPK) and other signalling pathways like STAT1 which are involved in immunogenic inflammation. Thus, synergistic stimulation of TAM by LPS and LDR revealed a major immunogenic mechanism of LDR in irradiated insulinomas. Therefore, targeting CD14 and associated signalling components like interleukin-1 receptor-associated kinase 4 (IRAK4), myeloid differentiation factor 88 (MyD88) may contribute in M1 retuning in large variety of tumors $(51,52)$. The translational impact of this concept was visualized upon immunization of mice carrying Panco2 tumor with DNA of attenuated strain of Salmonella typhimurium (as a natural source of TLR-4 ligand) which rejected implanted tumors (39). This was accompanied with drop in M2 TAM and VEGF titres and increase in $\mathrm{NO}$ titres demonstrating potential M1 retuning of TME in these mice. A similar shift in the phenotypic characteristics of TAM was reported by Kaimala et al. upon administration of attenuated Salmonella leading to functional maturation of intratumoral myeloid cells and enhancing the host's anti-tumor immune response (53). On a similar note, Lizotte et al. reported the therapeutic benefit of Listeria monocytogenes (L. mono) in ovarian cancer and showed that administration of attenuated $L$. mono triggered polarization of M2-TAM towards M1-like and contributed for tumor cell lysis via NOS2 production of nitric acid (54). Interestingly, using strains of Clostridium spp. to selectively colonize in TME is believed to contribute to TAM reprogramming $(55,56)$. Sfondrini et al. have shown the potent immunomodulatory effects of flagellin, the structural protein subunit of the bacterial flagellum, which signaling via TLR-5 decreases the abundance of $\mathrm{CD} 4^{+} \mathrm{CD} 25^{+}$ regulatory $\mathrm{T}$ cells (Tregs), which subsequently improves the anti-tumor response of the activated $\mathrm{CD}^{+} \mathrm{T}$ cells (57). Another novel treatment option is to engineer Clostridium acetobutylicum to express TNF- $\alpha$ that possess direct cytotoxic actions, as opposed to having pro-drug cleaving actions. The main mechanism by which these bacterial derived products depend upon their potential of enhancing the release of TNF- $\alpha$ that is strongly anti-tumor in nature (58). Additionally, these bacterial products can increase antitumor response of resident immune cells $(59,60)$. Duong et al. had summarized the potential benefits, challenges and ongoing clinical trials involving tumor-targeting bacteria (61). Systemic activation of innate immune cells through bacterial product, sometime manifest severe systemic side effects limiting their use as safe option for clinical intervention.

\section{Smac mimetic compounds: emerging neo adjuvant for TAM depolarization}

Intrinsic resistance for apoptosis is the first cardinal sign of exponentially growing tumors cells. During apoptotic cell death, caspases released from the mitochondrial innermembrane space inhibit the three inhibitors of apoptotic proteins (IAP) namely, cellular-IAP 1 and 2 and X-linked IAP and execute the process of apoptosis. However, it is evident that IAPs are overexpressed in many types of human cancers and is associated with chemoresistance, disease progression and poor prognosis in multiple cancer types (62). Among various tumor, pancreatic and lung adenocarcinoma tumor cells upregulate IAPs and survive radio and chemotherapy $(63,64)$. XIAP overexpression was found to shorten the survival of pancreatic cancer patients probably by modifying their resistance to apoptosis enhancing pancreatic carcinoma cells proliferative capacity. Apart from apoptosis, XIAP are also associated with lymphoproliferative syndrome (known as X-LP) and noncanonical NF- $\mathrm{kB}$ signaling which are also important for the chemo and radio resistance of these tumors. Therapeutic approach that results in the degradation of cIAPs and/ 
or antagonism of XIAP promote the lethal effects of $\mathrm{TNF} \alpha$ on cancer cells while shutting down the survival pathway mediated by $\mathrm{TNF} \alpha$ driven NF-kB expression. Increased expression of IAP/survivin proteins and immunological factors such as increased density of TAM contributes to increased resistance of pancreatic cancer to therapy. Moreover, a high degree of compensation among IAP proteins in tumor cells has shown to render treatment ineffective $(65,66)$. Therefore, pharmacological interventions alone may not serve the option for the management of resistant tumors and combinational approaches are likely favorable. For, e.g., TAM based approaches in conjunction of chemotherapy would be paramount for enhancing immune responses against tumors. In this context, we have recently demonstrated the use of Smac mimetic compounds (LC161) targeting IAP proteins on influencing the phenotypic and functional plasticity of macrophages (67), suggesting its potential as an adjuvant for activating TAM in solid tumors. The preclinical efficacy of LC161 has been reported with over-expression of XIAP and sensitizing osteosarcoma to TNF $\alpha$-mediated apoptosis (68). Lecis et al. also demonstrated that administration of smacmimetics was shown to induce a rapid inflammatory burst of TNF $\alpha$ and IFN $\gamma$ which resulted due to smac-mimetics effect of re-tuning TAM towards a proinflammatory M1like phenotype (69).

Smac mimetic-based disruption of macrophage plasticity enhances Th1 programming, effective clearance of tumor cells in the peripheral compartment (70), break resistance and enhance immunity in these cases. Thus smac-mimetics has tremendous potential for reconstituting immunity, promoting immunogenic death of tumor cells and their effective eradication which are decisive for tumor control. As successful induction of apoptosis using novel therapeutics may be a key strategy for preventing recurrence and metastasis, we believe that targeting IAP by Smac mimetic compounds which even had the potential to depolarize TAM towards anti-tumorigenic phenotype will be a good clinical strategy to fight against solid tumors.

\section{Nanoparticles: potent adjuvants for refractory M2 macrophages}

In the past decade, the growing evidences suggest that nanomedicine can be effectively utilized for activating newly generated macrophages along with existing refractor macrophages. These activated macrophages have a huge potential to fight against the latent infectious disease.
Nanoparticle based therapy can be efficiently utilized for regulating the differentiation of monocytes into iNOS positive M1 macrophages for substituting iNOS-/CD11b+/ Arg-1+ AAM during chronic infections. Recent studies from our group have displayed that biodegradable amino acid based pNAPA (71) and pLME (72) nanocapsule can potentially enhanced the differentiation of naïve macrophage towards the M1 effector phenotype. These data suggest that these nanocapsules may be used as an adjuvant for reactivating innate immunity for the management of infections as well as human malignancies. Nanoparticles represent an important delivery vehicle to deliver immune modulators/drugs/chemotherapeutic agents for rescuing the differentiation of effector phenotype of macrophage to refractory and/or reprogramming of refractory macrophages to effector macrophages. The delivery of specific siRNA nanoparticles (73) against CCR2 has been found to suppress the expression of CCR2 in monocytes and block the migration of monocytes to inflamed tissues. Treatment of lymphoma and colorectal xenografts using siCCR2 nanoparticles has been displayed to reduce the TAMs and CD11b+ cells as well as tumor size. Nanoparticle mediated strategies can be efficient for direct killing of the refractory macrophages. Biodegradability of nanocarriers is one of the crucial requirements to reduce the toxicity and side effect. Some examples are; biodegradable poly (ethylene glycol)-bpoly ( $\varepsilon$-caprolactone) (PEG-b-PCL), poly ( $\varepsilon$-caprolactone)b-poly (2-aminoethyl ethylene phosphate) (PCL-b-PPEEA), and PCL homopolymer. Nanoparticles loaded with CCL18 have been shown to silence CCL-18 in macrophages that lead to marked decrease in the M2 macrophages (74). The successful regulation of the macrophage behavior demonstrated herein shows great potential as an effective strategy for cancer therapy. For example, nanoliposomes are successfully used for the delivery of zoledronates and clodronates (bisphosphonates). The liposomes loaded with bisphosphonates were used for either subcutaneous or orthotropic injections in xenograft models and has been corelated with significant decrease in the AAM, angiogenesis and metastasis. Specific targeting is one of the major changes associated with nanocarriers/nanoparticles. This can be achieved by attaching either specific ligand including LyP1 and/or mannose receptors such as CD206 that are highly expressed on the surface of TAM/AAM for effective targeting of macrophages (75). Currently, PLA-PEG nanoparticles, liposomal formulations, and cyclodextrin nanoparticles are used for loading drugs, inhibitors plasmids, siRNAs such as sunitinib, TGF- $\beta$ 
inhibitors, IL-12 plasmids, CCR siRNA, VEGF siRNA for re-education of refractory macrophages for skewing in situ Th1 effector immune response against latent infections and human cancers $(76,77)$. Nanoparticles loaded with LR agonists represent an attractive approach to polarize AAM/ TAMs into M1 like macrophages (78). This has been shown that $\beta$-cyclodextrin nanoparticles loaded with R848 (agonist of TLR7/8) have guided superior drug delivery to TAMs in xenografts $(79,80)$. Also, $\beta$-cyclodextrin has been found to be associated with great drug (R848) solubilization, stability, re-education of TAMs to M1 phenotype and suppressed tumor growth. Another study displayed CD44 targeting hyaluronic acid-poly(ethylenimine) (HA-PEI)based nanoparticles for encapsulation of miR-125b.This report displayed that these nanoparticles specifically target TAMs in genetically engineered NSCLC murine model and significantly repolarize TAMs/AAM into M1 phenotype for anti-tumor immunotherapy (81).

\section{LnRNAs: emerging epigenetic modifiers for reprogramming of TAM in tumor patients}

LncRNA is one of the intrinsic non-cellular factor that plays an indispensable role in metastasis and progression (82). LncRNA is described as a large and diverse class of transcribed RNA molecules with a nucleotide length greater than 200 nucleotides that do not encode proteins $(83,84)$. Initially lncRNA was considered as "transcriptional noise", but recent advancements have demonstrated the role of lncRNA in the pathogenesis of many diseases (85). Furthermore, recent studies have shown a correlation amongst dysregulation of lncRNA expression with carcinogenesis, cell proliferation, drug resistance, and metastasis (86). We have recently proposed the role of IncRNA with a variety of cancers $(82-84,87)$. Similarly, a recent study has demonstrated that upregulation of LINC00662 is associated with increased cellular invasion and enhanced metastasis (88). This was accompanied with downregulation of WNT3A expression via competitive binding of lncRNA LINC00662 miR-15a, miR-16, and miR-107. Additionally, LINC00662 is capable of inducing $W n t / \beta$-catenin signaling in macrophages via paracrine manner; this activation signaling is important for polarization of M1 effector macrophages toward M2. Later LINC00662 mediated expression of WNT3A, Wnt/ $\beta$-catenin and M2 macrophage polarization was in HCC cell models as well. In same line several studies (89-92) were able to substantiate role of lncRNA dysregulation in various tumorigenic function of tumor-associated macrophages (Table 2). Although this is an enraging field however above studies provide ample evidence proving the association of lncRNA with the functions of tumor-associated macrophages. Thus, this field warrant further investigation and believe that $\operatorname{lnRNA}$ based analysis/intervention would be one of major therapeutic tool for modifying TAM polarization for therapy.

\section{Macrophages as palliatives agents for tissue reconstitution}

Successful therapy also requires vasculature normalization and homeostasis for normal physiological status. On the basis of regenerative potential of M2 or TAM macrophages these cells can potentially be targeting or enriched as palliative agent $(93,94)$. Although AAM resembles to TAM and secretes various growth factors which $(95,96)$ are sufficient to resolve acute/chronic inflammatory response which in manifested during/post therapy. On account of this, it is envisaged that these macrophages as whole cell infusion or their product can be artificially produced pharmaceutically for mitigation of toxicity related to chemo/radiotherapy in larger variety of cancer patients. M2 macrophages are known to promote activity of fibroblast/ stem cells which further warrant the significance of M2 or AAM macrophages in either palliative or regeneration medicine for variety of cancer or chronic inflammatory diseases like IBD where macrophage plasticity can dictate their pathologies.

\section{Conclusion and future perspectives}

Macrophages are heterogenous subset of immune cells within the TME and several recent studies have dissected their significant involvement in tumor progression, growth and metastasis. In this perspective review, we summarize significance of macrophage directed therapies for the management of tumor diseases emphasizing on the concept that change in macrophage index within the TME may afford therapeutic benefit in various tumordirected interventions, which may otherwise could be a major challenge. We summarized some of our recent findings that significantly contributed to the field of cancer immunotherapy. We believe a tremendous scope in use of Smac mimetics, polymer-based nanoparticles and lnRNA based interventions either alone or in conjunction with TAM based approaches will be highly beneficial for 
Table 2 Clinical association of lncRNA with various cancers and associated biological effect

\begin{tabular}{|c|c|c|c|c|c|c|c|}
\hline S.No. & Type of cancer & \multicolumn{2}{|c|}{ Total No. of patients } & $\begin{array}{r}\text { Type of } \\
\text { sample }\end{array}$ & $\begin{array}{l}\text { Effect on associated } \\
\text { IncRNA }\end{array}$ & Biological effect & Reference \\
\hline 1 & Gastric cancer & 30 & 30 & $\begin{array}{l}\text { Tumor } \\
\text { tissue }\end{array}$ & $\begin{array}{l}\text { Upregulation of IncRNA } \\
\text { ANCR }\end{array}$ & $\begin{array}{l}\text { (I) Increased tumor invasion; } \\
\text { (II) enhanced metastasis }\end{array}$ & (91) \\
\hline 2 & $\begin{array}{l}\text { Hepatocellular } \\
\text { carcinoma }\end{array}$ & 86 & - & $\begin{array}{l}\text { Tumor } \\
\text { tissue }\end{array}$ & $\begin{array}{l}\text { Upregulation of } \\
\text { LINC0066x2 }\end{array}$ & $\begin{array}{l}\text { (I) Enhanced cellular invasion; } \\
\text { (II) increased metastasis }\end{array}$ & $(90)$ \\
\hline 4 & $\begin{array}{l}\text { Hepatocellular } \\
\text { carcinoma }\end{array}$ & 20 & 20 & $\begin{array}{l}\text { Tumor } \\
\text { tissue }\end{array}$ & $\begin{array}{l}\text { Upregulation of } \\
\text { ELMOI-ASI }\end{array}$ & $\begin{array}{l}\text { (I) Promote angiogenesis; (II) increase the } \\
\text { immunosuppressive properties in cancer } \\
\text { cells }\end{array}$ & (93) \\
\hline
\end{tabular}

enhancing protective immunity against refractory tumors. Such combinational approach has not been explored so far and hold tremendous potential in clinics for interventions.

\section{Acknowledgments}

Funding: This work was supported by Ramanujan grant (SR/S2/RJN/03/2012) to HP. VN was supported by LSRB grant (LSRB-271/SH\&DD/2015). TSB was supported by Indo Russian grant (INT/RUS/RFBR/P-311) to AJ. S.K. acknowledge the funding support from SERB (ECR/001173/2016) and MG received funding from DBT Ramalinghaswamy grant (BT/RLF/Re-entry/24/2014).

\section{Footnote}

Provenance and Peer Review: This article was commissioned by the Guest Editor (Gaurav Pandey) for the series "Tumor Associated Macrophages in Solid Tumor: Friend or Foe" published in Annals of Translational Medicine. The article was sent for external peer review organized by the Guest Editor and the editorial office.

Conflicts of Interest: All authors have completed the ICMJE uniform disclosure form (available at http://dx.doi. org/10.21037/atm-20-695). The series "Tumor Associated Macrophages in Solid Tumor: Friend or Foe" was commissioned by the editorial office without any funding or sponsorship. The authors have no other conflicts of interest to declare.
Ethical Statement: The authors are accountable for all aspects of the work in ensuring that questions related to the accuracy or integrity of any part of the work are appropriately investigated and resolved.

Open Access Statement: This is an Open Access article distributed in accordance with the Creative Commons Attribution-NonCommercial-NoDerivs 4.0 International License (CC BY-NC-ND 4.0), which permits the noncommercial replication and distribution of the article with the strict proviso that no changes or edits are made and the original work is properly cited (including links to both the formal publication through the relevant DOI and the license). See: https://creativecommons.org/licenses/by-nc-nd/4.0/.

\section{References}

1. Smyth MJ, Ngiow SF, Ribas A, et al. Combination cancer immunotherapies tailored to the tumour microenvironment. Nat Rev Clin Oncol 2016;13:143-58.

2. McAllister SS, Weinberg RA. The tumour-induced systemic environment as a critical regulator of cancer progression and metastasis. Nat Cell Biol 2014;16:717-27.

3. Wang M, Zhao J, Zhang L, et al. Role of tumor microenvironment in tumorigenesis. J Cancer 2017;8:761-73.

4. Hinshaw DC, Shevde LA. The Tumor Microenvironment Innately Modulates Cancer Progression. Cancer Res 2019;79:4557-66.

5. Guerriero JL. Macrophages: The Road Less Traveled, 


\section{Page 10 of 13}

Changing Anticancer Therapy. Trends Mol Med 2018;24:472-89.

6. Dehne N, Mora J, Namgaladze D, et al. Cancer cell and macrophage cross-talk in the tumor microenvironment. Curr Opin Pharmacol 2017;35:12-9.

7. Gentles AJ, Newman AM, Liu CL, et al. The prognostic landscape of genes and infiltrating immune cells across human cancers. Nat Med 2015;21:938-45.

8. Wynn TA, Chawla A, Pollard JW. Macrophage biology in development, homeostasis and disease. Nature 2013;496:445-55.

9. Laviron M, Boissonnas A. Ontogeny of Tumor-Associated Macrophages. Front Immunol 2019;10:1799.

10. Mantovani A, Locati M. Tumor-associated macrophages as a paradigm of macrophage plasticity, diversity, and polarization: lessons and open questions. Arterioscler Thromb Vasc Biol 2013;33:1478-83.

11. Jeannin P, Paolini L, Adam C, et al. The roles of CSFs on the functional polarization of tumor-associated macrophages. FEBS J 2018;285:680-99.

12. Novak ML, Koh TJ. Macrophage phenotypes during tissue repair. J Leukoc Biol 2013;93:875-81.

13. Biswas SK, Mantovani A. Macrophage plasticity and interaction with lymphocyte subsets: cancer as a paradigm. Nat Immunol 2010;11:889-96.

14. Farkona S, Diamandis EP, Blasutig IM. Cancer immunotherapy: the beginning of the end of cancer? BMC Med 2016;14:73.

15. Klug F, Prakash H, Huber PE, et al. Low-dose irradiation programs macrophage differentiation to an iNOS(+)/ M1 phenotype that orchestrates effective T cell immunotherapy. Cancer Cell 2013;24:589-602.

16. Mantovani A, Marchesi F, Malesci A, et al. Tumourassociated macrophages as treatment targets in oncology. Nat Rev Clin Oncol 2017;14:399-416.

17. van Dalen FJ, van Stevendaal M, Fennemann FL, et al. Molecular Repolarisation of Tumour-Associated Macrophages. Molecules 2018;24:9.

18. Sica A, Bronte V. Altered macrophage differentiation and immune dysfunction in tumor development. J Clin Invest 2007;117:1155-66.

19. Paluskievicz CM, Cao X, Abdi R, et al. T Regulatory Cells and Priming the Suppressive Tumor Microenvironment. Front Immunol 2019;10:2453.

20. Idikio HA. Human cancer classification: a systems biologybased model integrating morphology, cancer stem cells, proteomics, and genomics. J Cancer 2011;2:107-15.

21. Leclerc M, Voilin E, Gros G, et al. Regulation of

\section{Nadella et al. Macrophage are paramount for cancer therapy}

antitumour CD8 T-cell immunity and checkpoint blockade immunotherapy by Neuropilin-1. Nat Commun 2019;10:3345.

22. Rowe DH, Huang J, Kayton ML, et al. Anti-VEGF antibody suppresses primary tumor growth and metastasis in an experimental model of Wilms' tumor. J Pediatr Surg 2000;35:30-2; discussion 32-3.

23. Cannarile MA, Weisser M, Jacob W, et al. Colonystimulating factor 1 receptor (CSF1R) inhibitors in cancer therapy. J Immunother Cancer 2017;5:53.

24. Mazzieri R, Pucci F, Moi D, et al. Targeting the ANG2/ TIE2 axis inhibits tumor growth and metastasis by impairing angiogenesis and disabling rebounds of proangiogenic myeloid cells. Cancer Cell 2011;19:512-26.

25. Harada H, Kizaka-Kondoh S, Li G, et al. Significance of $\mathrm{HIF}-1$-active cells in angiogenesis and radioresistance. Oncogene 2007;26:7508-16.

26. Palazon A, Tyrakis PA, Macias D, et al. An HIF-1alpha/ VEGF-A Axis in Cytotoxic T Cells Regulates Tumor Progression. Cancer Cell 2017;32:669-83.e5.

27. Wei C, Yang C, Wang S, et al. Crosstalk between cancer cells and tumor associated macrophages is required for mesenchymal circulating tumor cell-mediated colorectal cancer metastasis. Mol Cancer 2019;18:64.

28. Steenbrugge J, Breyne K, Demeyere K, et al. Antiinflammatory signaling by mammary tumor cells mediates prometastatic macrophage polarization in an innovative intraductal mouse model for triple-negative breast cancer. J Exp Clin Cancer Res 2018;37:191.

29. Noy R, Pollard JW. Tumor-associated macrophages: from mechanisms to therapy. Immunity 2014;41:49-61.

30. Lim SY, Yuzhalin AE, Gordon-Weeks AN, et al. Tumor-infiltrating monocytes/macrophages promote tumor invasion and migration by upregulating S100A8 and S100A9 expression in cancer cells. Oncogene 2016;35:5735-45.

31. Yang Y, Sun M, Wang L, Jiao B. HIFs, angiogenesis, and cancer. J Cell Biochem 2013;114:967-74.

32. Blériot C, Ginhoux F. Macrophage depletion in cancer therapy: A double-edged sword. Cell Immunol 2018;331:178-9.

33. Ries CH, Cannarile MA, Hoves S, et al. Targeting tumorassociated macrophages with anti-CSF-1R antibody reveals a strategy for cancer therapy. Cancer Cell 2014;25:846-59.

34. Peranzoni E, Lemoine J, Vimeux L, et al. Macrophages impede CD8 T cells from reaching tumor cells and limit the efficacy of anti-PD-1 treatment. Proc Natl Acad Sci U S A 2018;115:E4041-50. 
35. Duong L, Radley-Crabb HG, Gardner JK, et al. Macrophage Depletion in Elderly Mice Improves Response to Tumor Immunotherapy, Increases Anti-tumor T Cell Activity and Reduces Treatment-Induced Cachexia. Front Genet 2018;9:526.

36. Bader JE, Enos RT, Velazquez KT, et al. Macrophage depletion using clodronate liposomes decreases tumorigenesis and alters gut microbiota in the AOM/DSS mouse model of colon cancer. Am J Physiol Gastrointest Liver Physiol 2018;314:G22-31.

37. Wu X, Schulte BC, Zhou Y, et al. Depletion of M2like tumor-associated macrophages delays cutaneous T-cell lymphoma development in vivo. J Invest Dermatol 2014;134:2814-22.

38. Sessa A, Battini G, Meroni M, et al. Multifocal bilateral renal cell carcinoma and retinal angiomas in a patient with de novo von Hippel-Lindau disease: identification of a new germline mutation. J Nephrol 2005;18:209-12.

39. Prakash H, Klug F, Nadella V,et al. L. Low doses of gamma irradiation potentially modifies immunosuppressive tumor microenvironment by retuning tumor-associated macrophages: lesson from insulinoma. Carcinogenesis 2016;37:301-13.

40. Nadella V, Singh S, Jain A, et al. Low dose radiation primed iNOS + M1macrophages modulate angiogenic programming of tumor derived endothelium. Mol Carcinog 2018;57:1664-71.

41. Liu J, Zhou J, Wu M, et al. Low-Dose Total Body Irradiation Can Enhance Systemic Immune Related Response Induced by Hypo-Fractionated Radiation. Front Immunol 2019;10:317.

42. Menon H, Chen D, Ramapriyan R, et al. Influence of lowdose radiation on abscopal responses in patients receiving high-dose radiation and immunotherapy. J Immunother Cancer 2019;7:237.

43. Arnold SM, Kudrimoti M, Dressler EV, et al. Using low-dose radiation to potentiate the effect of induction chemotherapy in head and neck cancer: Results of a prospective phase 2 trial. Adv Radiat Oncol 2016;1:252-9.

44. Dey S, Spring PM, Arnold S, et al. Low-dose fractionated radiation potentiates the effects of Paclitaxel in wildtype and mutant p53 head and neck tumor cell lines. Clin Cancer Res 2003;9:1557-65.

45. Lapteva N, Seethammagari MR, Hanks BA, et al. Enhanced activation of human dendritic cells by inducible CD40 and Toll-like receptor-4 ligation. Cancer Res 2007;67:10528-37.

46. Nierkens S, den Brok MH, Garcia Z, et al. Immune adjuvant efficacy of $\mathrm{CpG}$ oligonucleotide in cancer treatment is founded specifically upon TLR9 function in plasmacytoid dendritic cells. Cancer Res 2011;71:6428-37.

47. Kim BS, Spinner DS, Kascsak RJ, et al. Inflammatory mediators are inhibited by a taurine metabolite in CpG oligodeoxynucleotide and IFN-r activated macrophage cell line. J Drugs Dermatol 2013;12:551-7.

48. Basith S, Manavalan B, Yoo TH, et al. Roles of toll-like receptors in cancer: a double-edged sword for defense and offense. Arch Pharm Res 2012;35:1297-316.

49. Sato Y, Goto Y, Narita N, et al. Cancer Cells Expressing Toll-like Receptors and the Tumor Microenvironment. Cancer Microenviron 2009;2 Suppl 1:205-14.

50. Tsan MF. Toll-like receptors, inflammation and cancer. Semin Cancer Biol 2006;16:32-7.

51. Islam MA, Proll M, Holker M, et al. Alveolar macrophage phagocytic activity is enhanced with LPS priming, and combined stimulation of LPS and lipoteichoic acid synergistically induce pro-inflammatory cytokines in pigs. Innate Immun 2013;19:631-43.

52. Tachado SD, Li X, Bole M, et al. MyD88-dependent TLR4 signaling is selectively impaired in alveolar macrophages from asymptomatic $\mathrm{HIV}+$ persons. Blood 2010;115:3606-15.

53. Kaimala S, Mohamed YA, Nader N, et al. Salmonellamediated tumor regression involves targeting of tumor myeloid suppressor cells causing a shift to M1-like phenotype and reduction in suppressive capacity. Cancer Immunol Immunother 2014;63:587-99.

54. Lizotte PH, Baird JR, Stevens CA, et al. Attenuated Listeria monocytogenes reprograms M2-polarized tumor-associated macrophages in ovarian cancer leading to iNOS-mediated tumor cell lysis. Oncoimmunology 2014;3:e28926.

55. Kubiak AM, Minton NP. The potential of clostridial spores as therapeutic delivery vehicles in tumour therapy. Res Microbiol 2015;166:244-54.

56. Umer B, Good D, Anne J, et al. Clostridial spores for cancer therapy: targeting solid tumour microenvironment. J Toxicol 2012;2012:862764.

57. Sfondrini L, Rossini A, Besusso D, et al. Antitumor activity of the TLR-5 ligand flagellin in mouse models of cancer. J Immunol 2006;176:6624-30.

58. Theys J, Nuyts S, Landuyt W, et al. Stable Escherichia coli-Clostridium acetobutylicum shuttle vector for secretion of murine tumor necrosis factor alpha. Appl Environ Microbiol 1999;65:4295-300.

59. Brunet AS, Ploton C, Galambrun C, et al. Low incidence 
of sepsis due to viridans streptococci in a ten-year retrospective study of pediatric acute myeloid leukemia. Pediatr Blood Cancer 2006;47:765-72.

60. Barbé S, Van Mellaert L, Theys J, et al. Secretory production of biologically active rat interleukin-2 by Clostridium acetobutylicum DSM792 as a tool for antitumor treatment. FEMS Microbiol Lett 2005;246:67-73.

61. Duong MT, Qin Y, You SH, et al. Bacteria-cancer interactions: bacteria-based cancer therapy. Exp Mol Med 2019;51:1-15.

62. Tamm I, Kornblau SM, Segall H, et al. Expression and prognostic significance of IAP-family genes in human cancers and myeloid leukemias. Clin Cancer Res 2000;6:1796-803.

63. Krepela E, Dankova P, Moravcikova E, et al. Increased expression of inhibitor of apoptosis proteins, survivin and XIAP, in non-small cell lung carcinoma. Int J Oncol 2009;35:1449-62.

64. Li S, Sun J, Yang J, et al. XIAP expression is associated with pancreatic carcinoma outcome. Mol Clin Oncol 2013;1:305-8.

65. Saio M, Radoja S, Marino M, et al. Tumor-infiltrating macrophages induce apoptosis in activated CD8(+) $\mathrm{T}$ cells by a mechanism requiring cell contact and mediated by both the cell-associated form of TNF and nitric oxide. J Immunol 2001;167:5583-93.

66. Pollard JW. Tumour-educated macrophages promote tumour progression and metastasis. Nat Rev Cancer 2004;4:71-8.

67. Nadella V, Mohanty A, Sharma L, et al. Inhibitors of Apoptosis Protein Antagonists (Smac Mimetic Compounds) Control Polarization of Macrophages during Microbial Challenge and Sterile Inflammatory Responses. Front Immunol 2018;8:1792.

68. Infante JR, Dees EC, Olszanski AJ, et al. Phase I doseescalation study of LCL161, an oral inhibitor of apoptosis proteins inhibitor, in patients with advanced solid tumors. J Clin Oncol 2014;32:3103-10.

69. Lecis D, De Cesare M, Perego P, et al. Smac mimetics induce inflammation and necrotic tumour cell death by modulating macrophage activity. Cell Death Dis. 2013;4:e920.

70. Chesi M, Mirza NN, Garbitt VM, et al. IAP antagonists induce anti-tumor immunity in multiple myeloma. Nat Med 2016;22:1411-20.

71. Yamala AK, Nadella V, Mastai Y, et al. Poly-N-acryloyl(l-phenylalanine methyl ester) hollow core nanocapsules facilitate sustained delivery of immunomodulatory drugs and exhibit adjuvant properties. Nanoscale 2017;9:14006-14.

72. Yamala AK, Nadella V, Mastai Y, et al. P-LME polymer nanocapsules stimulate naive macrophages and protect them from oxidative damage during controlled drug release. J Appl Polym Sci 2020;137:48363.

73. Leuschner F, Dutta P, Gorbatov R, et al. Therapeutic siRNA silencing in inflammatory monocytes in mice. Nat Biotechnol 2011;29:1005-10.

74. Liang S, Zheng J, Wu W, et al. A Robust Nanoparticle Platform for RNA Interference in Macrophages to Suppress Tumor Cell Migration. Front Pharmacol 2018;9:1465.

75. Yan Z, Wang F, Wen Z, et al. LyP-1-conjugated PEGylated liposomes: a carrier system for targeted therapy of lymphatic metastatic tumor. J Control Release 2012;157:118-25.

76. Liu X, Gao X, Zheng S, et al. Modified nanoparticle mediated IL-12 immunogene therapy for colon cancer. Nanomedicine 2017;13:1993-2004.

77. Xu Z, Wang Y, Zhang L, et al. Nanoparticle-delivered transforming growth factor-beta siRNA enhances vaccination against advanced melanoma by modifying tumor microenvironment. ACS Nano 2014;8:3636-45.

78. Shime H, Matsumoto M, Oshiumi H, et al. Toll-like receptor 3 signaling converts tumor-supporting myeloid cells to tumoricidal effectors. Proc Natl Acad Sci U S A 2012;109:2066-71.

79. Rodell CB, Arlauckas SP, Cuccarese MF, et al. TLR7/8agonist-loaded nanoparticles promote the polarization of tumour-associated macrophages to enhance cancer immunotherapy. Nat Biomed Eng 2018;2:578-88.

80. Cully M. Cancer: Re-educating tumour-associated macrophages with nanoparticles. Nat Rev Drug Discov 2018;17:468.

81. Parayath NN, Parikh A, Amiji MM. Repolarization of Tumor-Associated Macrophages in a Genetically Engineered Nonsmall Cell Lung Cancer Model by Intraperitoneal Administration of Hyaluronic Acid-Based Nanoparticles Encapsulating MicroRNA-125b. Nano Lett 2018;18:3571-9.

82. Khandelwal A, Bacolla A, Vasquez KM, et al. Long noncoding RNA: A new paradigm for lung cancer. Mol Carcinog 2015;54:1235-51.

83. Tamang S, Acharya V, Roy D, et al. SNHG12: An LncRNA as a Potential Therapeutic Target and Biomarker for Human Cancer. Front Oncol 2019;9:901.

84. Malhotra A, Jain M, Prakash H, et al. The regulatory roles of long non-coding RNAs in the development 
of chemoresistance in breast cancer. Oncotarget 2017;8:110671-84.

85. Guttman M, Donaghey J, Carey BW, et al. lincRNAs act in the circuitry controlling pluripotency and differentiation. Nature 2011;477:295-300.

86. Chen DL, Chen LZ, Lu YX, et al. Long noncoding RNA XIST expedites metastasis and modulates epithelialmesenchymal transition in colorectal cancer. Cell Death Dis 2017;8:e3011.

87. Saluja R, Kumar A, Jain M, et al. Role of Sphingosine1-Phosphate in Mast Cell Functions and Asthma and Its Regulation by Non-Coding RNA. Front Immunol 2017;8:587.

88. Tian X, Wu Y, Yang Y, et al. Long noncoding RNA LINC00662 promotes M2 macrophage polarization and hepatocellular carcinoma progression via activating Wnt/ beta-catenin signaling. Mol Oncol 2020;14:462-483.

89. Xie C, Guo Y, Lou S. LncRNA ANCR Promotes Invasion and Migration of Gastric Cancer by Regulating FoxO1 Expression to Inhibit Macrophage M1 Polarization. Dig Dis Sci 2020. [Epub ahead of print].

90. Ye Y, Guo J, Xiao P, et al. Macrophages-induced long noncoding RNA H19 up-regulation triggers and activates the miR-193b/MAPK1 axis and promotes cell aggressiveness in hepatocellular carcinoma. Cancer Lett

Cite this article as: Nadella V, Garg M, Kapoor S, Barwal TS, Jain A, Prakash H. Emerging neo adjuvants for harnessing therapeutic potential of M1 tumor associated macrophages (TAM) against solid tumors: Enusage of plasticity. Ann Transl Med 2020;8(16):1029. doi: 10.21037/atm-20-695
2020;469:310-22.

91. Sun Y, Xu J. TCF-4 Regulated lncRNA-XIST Promotes M2 Polarization Of Macrophages And Is Associated With Lung Cancer. Onco Targets Ther 2019;12:8055-62.

92. Luo T, Chen M, Zhao Y, et al. Macrophage-associated lncRNA ELMO1-AS1: a novel therapeutic target and prognostic biomarker for hepatocellular carcinoma. Onco Targets Ther 2019;12:6203-16.

93. Kim JW, Ha KY, Molon JN, et al. Bone marrow-derived mesenchymal stem cell transplantation for chronic spinal cord injury in rats: comparative study between intralesional and intravenous transplantation. Spine (Phila $\mathrm{Pa}$ 1976) 2013;38:E1065-74.

94. Hoegl S, Zwissler B, Eltzschig HK, et al. Acute respiratory distress syndrome following cardiovascular surgery: current concepts and novel therapeutic approaches. Curr Opin Anaesthesiol 2016;29:94-100.

95. Broughton BR, Lim R, Arumugam TV, et al. Post-stroke inflammation and the potential efficacy of novel stem cell therapies: focus on amnion epithelial cells. Front Cell Neurosci 2013;6:66.

96. Boxman IL, Ruwhof C, Boerman OC, et al. Role of fibroblasts in the regulation of proinflammatory interleukin IL-1, IL-6 and IL-8 levels induced by keratinocyte-derived IL-1. Arch Dermatol Res 1996;288:391-8. 